\section{Re: Burnout in Young Family Physicians: Variation Across States}

I read with great interest the article of Hansen et $\mathrm{al}^{1}$ in a recent issue of the journal. The authors performed a cross-sectional study to determine the rates of emotional exhaustion and depersonalization and symptoms of burnout in family physicians 3 years out of training. They report high rates of burnout, with considerable variation between states. The authors should be congratulated for performing a study in an important topic in medical practice. ${ }^{2,3}$ Moreover, the need to identify specific geographical locations associated with high burnout rates in physicians is an innovative concept that needs to be further explored.,

Although the study of Hansen et $\mathrm{al}^{1}$ was well conducted, there are some questions regarding the study that need to be clarified. The authors have chosen to exclude the personal accomplishment components of the Maslach burnout survey. The personal accomplishment assessment has been shown to be protective against burnout among high-achiever professionals. In addition, several confounding factors associated with burnout (eg, control over professional life, working hours) were not investigated by the authors and could confound the variance observed by the authors across states. Lastly, it would be important to evaluate response bias particularly in states with less than $60 \%$ response rate to determine the generalizability of the study results.

I would welcome some comments to address the aforementioned issues, as they were not discussed by the authors. This would further support the findings of this important study.

Mark C. Kendall, MD

Department of Anesthesiology, Rhode Island Hospital The Warren Alpert Medical School of Brown University

Providence, Rhode Island

To see this article online, please go to: http://jabfm.org/content/ 31/4/663.full.

\section{References}

1. Hansen A, Peterson LE, Fang B, Phillips RL Jr. Burnout in young family physicians: variation across states. J Am Board Fam Med 2018;31:7-8.
2. De Oliveira GS Jr. Biological evidence of the impact of burnout on the health of anesthesiologists. J Clin Anesth 2017;4:62.

3. Puffer JC, Knight HC, O’Neill TR, Rassolian M, Bazemore AW, Peterson LE, Baxley EG. Prevalence of burnout in board certified family physicians. J Am Board Fam Med 2017;30:125-26.

4. Vinson AE, Zurakowski D, Randel GI, Schlecht KD. National survey of US academic anesthesiology chairs on clinician wellness. J Clin Anesth 2016;34:623-31.

5. Gonzalez LS, Donnelly MJ. A survey of residency program directors in anesthesiology regarding mentorship of residents. J Clin Anesth 2016;33:254-65.

doi: 10.3122/jabfm.2018.04.180076

\section{Response: Re: Burnout in Young Family Physicians: Variation Across States (J Am Board Fam Med 2018;31:663-664.)}

Dr. Kendall brings up valuable considerations as we seek to understand the personal and institutional factors influencing physician burnout. As Dr. Mack discussed in his commentary ${ }^{1}$, family physicians experience burnout at rates that are higher than average for many reasons, including less time spent in direct patient care, more administrative burdens, and more work hours. ${ }^{2}$ Relevant policies and culture in family medicine and health care vary at the state level, allowing states to function as real-life laboratories; understanding state-level variation can help us to identify and remedy the underlying causes of burnout. Dr. Kendall offers 3 major criticisms of our study that are largely beyond the scope of our study but suggest avenues for future research.

Dr. Kendall notes that our analysis did not include the personal accomplishment component of the Maslach Burnout Inventory. The National Graduate Survey includes only 2 validated questions measuring the emotional exhaustion and depersonalization domains, and it does not include a question measuring personal accomplishment. The survey does include a 3-part question about satisfaction with training and the profession, and a 6-part question about satisfaction with current principal practice. While not a perfect substitute for the full Maslach Burnout Inventory, these data may be worth examining to identify state-level variation in future work. However, emotional exhaus- 
Table 1. Characteristics of Respondents versus Nonrespondents for 2013 Family Medicine Residency Graduates from the 2016 National Family Medicine Graduate Survey $(\mathrm{N}=3063)$

\begin{tabular}{lccr}
\hline Characteristic & Respondents $(\mathrm{n}=2,069)$ & Nonrespondents $(\mathrm{n}=994)$ & $P$ value \\
\hline MD & $1767(85.4)$ & $828(83.3)$ & .13 \\
International medical graduate & $762(36.8)$ & $398(40.0)$ & .09 \\
Female sex & $1169(56.5)$ & $528(53.1)$ & .08 \\
Age (years), mean \pm SD & $36.1 \pm 4.5$ & $36.4 \pm 4.6$ & .06 \\
\hline
\end{tabular}

Data are $\mathrm{n}(\%)$ unless otherwise indicated.

$\mathrm{SD}$, standard deviation.

tion and depersonalization are the questions with the highest loading factor for measuring burnout, and using these 2 single-item measures provides a meaningful assessment of burnout. ${ }^{3}$

Dr. Kendall expresses concerns about unmeasured confounding in our descriptive analysis from factors associated with burnout, including professional life and working hours. Prior research on family physicians found such variables to be predictive of burnout. ${ }^{4}$ However, a full regression analysis is beyond the scope of a policy brief, and we doubt that any amount of statistical adjustment will close a $30 \%$ difference in emotional exhaustion rates between states.

Dr. Kendall also wonders about response bias in our results. No systematic differences were found between respondents and nonrespondents to the 2016 National Graduate Survey by age, sex, degree type, and US versus international medical school (Table 1). Given that the response rate by state in our sample ranged from $55.7 \%$ to $78.6 \%$, we have no reason to believe there was a state-level systematic selection bias.

We appreciate the attention physician wellness and burnout are receiving and believe this is an important area of research. We welcome more studies about the distribution of burnout and its causes to build a better family physician and health care workforce.

Anna Hansen, BA Department of Sociology and College of Medicine University of Kentucky Lexington, KY Elizabeth Rose Hansen, BA Lars E. Peterson, MD, PhD American Board of Family Medicine Lexington, KY lpeterson@theabfm.org

To see this article online, please go to: http://jabfm.org/content/ 31/4/664.full.

\section{References}

1. Mack DO. A state chapter perspective on burnout and resiliency. J Am Board Fam Med 2018;31:5-6.

2. Friedberg MW, Chen PG, Van Busum KR, et al. Factors affecting physician professional satisfaction and their implications for patient care, health systems, and health policy. Rand Health Q 2014;3:1.

3. West CP, Dyrbye LN, Sloan JA, Shanafelt TD. Single item measures of emotional exhaustion and depersonalization are useful for assessing burnout in medical professionals. J Gen Intern Med 2009;24:1318-21.

4. Rassolian M, Peterson LE, Fang B, et al. Workplace factors associated with burnout of family physicians. JAMA Intern Med 2017;177:1036-8.

doi: 10.3122/jabfm.2018.04.180137 